\title{
Research Paper \\ Antibiotic Susceptibility Profile of Clostridium Difficile Bacteria Isolated from Older Residents of a Nursing Home in Iran
}

\author{
Ahmad Ghasemi $^{1}$ (D) , Ashraf Mohabati Mobarez ${ }^{1}$ (D), Ehsan Mostafavi ${ }^{2,3}$ (i) \\ 1. Department of Bacteriology, Faculty of Medical Sciences, Tarbiat Modares University, Tehran, Iran. \\ 2. Department of Epidemiology and Biostatics, Research Centre for Emerging and Reemerging Infectious Diseases, Pasteur Institute of Iran, Tehran, Iran. \\ 3. Laboratory Reference Country Plague, Tolarmi and Q Fever, Research Centre for Emerging and Reemerging Infectious Diseases Pasteur Institute of Iran, Kabudar \\ Ahang, Hamadan, Iran.
}

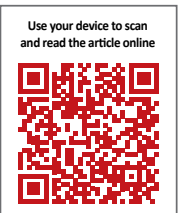

Chtertion: Ghasemi A, Mobarez Mohabati A, Mostafavi E. [Antibiotic Susceptibility Profile of Clostridium Difficile Bacteria Isolated from Older Residents of a Nursing Home in Iran (Persian)]. Iranian Journal of Ageing. 2021; 15(4):496-505. https://doi. org/10.32598/sija.15.4.2997

doi https://doi.org/10.32598/sija.15.4.2997

\section{(c) (1) (5)}

Received: 01 Jul 2020 Accepted: 31 Oct 2020 Available Online: 24 Feb 2021

Keywords:

Clostridium difficile, Older adults, Antibiotic susceptibility

\section{A B STRACT}

Objectives Clostridium difficile (C. diff) is a gram-positive anaerobic bacterium knwon as the most common cause of nosocomial diarrhea in nursing homes. The antibiotic susceptibility profile is the basic way for successful treatment due to antimicrobial resistance. This present study aims to assess the antibiotic susceptibility profile of $\mathrm{C}$. diff isolated from older residents of a nursing home in Tehran, Iran.

Methods \& Materials Forty-two isolates of C. diff were used in this study collected from 289 residents of Kahrizak Nursing Home. Antibiotic susceptibility testing was conducted by using disk-diffusion method, agar dilution method, and Epsilometer test (E-test).

Results All C. diff strains were susceptible to Metronidazole, Vancomycin, Rifampicin, Linezolid and Tigecycline. By using the disk-diffusion method, the highest rate of resistance was related to Clindamycin (100\%), Levofloxacin (96.2\%), Imipenem (81\%), Azithromycin (61\%) and Erythromycin (54.8\%). All C. diff strains were susceptible to Metronidazole under E-test. Furthermore, $100 \%$ and $59.5 \%$ of strains were susceptible to Vancomycin and Erythromycin, respectively under agar dilution test.

Conclusion C. diff strains are sensitive to Vancomycin and Metronidazole. These two antibiotics can be used to treat $\mathrm{C}$. diff infections in older adults. The disk diffusion method can be used as a screening test to determine antibiotic resistance.

\section{Extended Abstract}

\section{Introduction}

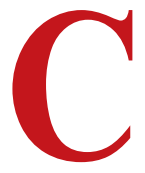

lostridium difficile (C. diff) is a gram-positive, obligate anaerobic, and spore-forming bacillus that was identified in 1935 as part of the flora of healthy infants. This bacterium is an important human pathogen that has been introduced as a causative agent of antibiotic-associated diarrhea and pseudomembranous colitis $[1,2]$. This bacterium is the most common cause of infectious diarrhea in nursing homes, which in recent years. About 3 million cases of diarrhea and colitis are reported annually. Mortality associated with $\mathrm{C}$. diff infectious diarrhea is estimated at $17 \%$, which is higher in the elderly $[5,6]$. Colonization by $\mathrm{C}$. diff isolates producing toxins has been reported in nursing home residents ten times more than in those dwelling in the community [7]. It is reported that about $57 \%$ of the residents of nursing homes can be carriers of this bacterium $[7,8]$. In Iran, there is also the problem of irrational use or overuse of antibiotics [14]. The resistance to these

* Corresponding Author:

Ashraf Mobarez Mohabati, PhD.

Address: Department of Bacteriology, Faculty of Medical Sciences, Tarbiat Modares University, Tehran, Iran. Tel: +98 (21) 82883862

E-mail: mmmobarez@modares.ac.ir 
drugs is increasing in the world and there are no reports of their usage among the residents of nursing homes in Iran. Antibiotic susceptibility profile is the most basic way for successful treatment due to antimicrobial resistance. This study aims to evaluate the antibiotic susceptibility profiles of C. diff bacteria isolated from the elderly living in a nursing home in Tehran, Iran.

\section{Methods \& Materials}

In this study, 289 fecal samples from residents of Kahrizak nursing home in Tehran, Iran who were over 60 years old (ranging from 60 to 101) and hospitalized for at least one month were examined for antibiotic susceptibility (44.3\% male and 55.7\% female). of 289 samples, 42 (14.5\%) tested positive for C. diff.

Antibiotic susceptibility test by disk diffusion method

Discs of antibiotics were metronidazole, vancomycin, imipenem, clindamycin, chloramphenicol, azithromycin, linezolid, tigecycline, teicoplanin, rifampin, erythromycin and amoxicillin-clavulanic acid (Mast Group Ltd, UK). Isolates were first cultured in Cycloserine-cefoxitin fructose Agar (CCFA) medium and $1 \mathrm{McF}$ arland dilution in physiological saline was prepared from each grown sample. Then, swab was used for superficial culture on Bru- cella agar medium (Merck Co.) with 5\% sheep blood and 1 $\mu \mathrm{g} / \mathrm{mL}$ vitamin $\mathrm{K}$ (Sigma Co.). The tested disks were then placed on the culture medium and incubated for 48 hours at $37^{\circ} \mathrm{C}$ in an anaerobic jar containing GasPak type A(Merck Co.). Disk diffusion of each drug was interpreted according to published articles [15-21].

Antibiotic susceptibility test by agar dilution method and E-test

Antibiotic susceptibility testing was performed with agar dilution method for vancomycin and erythromycin antibiotics (Sigma Co.) and Epsilometer test (E-test) for metronidazole antibiotic (Liofilchem Co., Italy), according to the protocol of Clinical and Laboratory Standards Institute 2018 (CLSI 2018). First, 0.5 McFarland dilution was prepared from isolates grown in CCFA culture medium. Then, 1 microliter of $0.5 \mathrm{McF}$ arland dilution was cultured on Brucella agar medium with different dilutions of antibiotics, containing 5\% sheep blood and vitamin $\mathrm{K}(1 \mu \mathrm{g} / \mathrm{mL})$. For E-test, $1 \mathrm{McF}$ arland dilution was cultured on by swap on Brucella agar medium containing 5\% sheep blood and $1 \mu \mathrm{g} / \mathrm{mL}$ vitamin $\mathrm{K}$. Next, metronidazole strip was placed on the culture. The cultured plates were then incubated for 48 hours at $37^{\circ} \mathrm{C}$ in an anaerobic jar containing GasPak type A [22]. The results were interpreted according to CLSI 2018 guidelines.

Table 1. Prevalence of antibiotic susceptibility by disk diffusion method in C. diff isolates

\begin{tabular}{|c|c|c|c|}
\hline Antibiotic Disk & Susceptible No. (\%) & Resistant, No. (\%) & Range zone, $\mathrm{mm}$ \\
\hline Clindamycin $2 \mu \mathrm{g}$ & $0(0.00)$ & $42(100)$ & 0 \\
\hline Teicoplanin 30 $\mathrm{\mu g}$ & $40(95.2)$ & $2(4.8)$ & $16-32$ \\
\hline Tigecycline $15 \mu \mathrm{g}$ & $42(100)$ & $0(0.00)$ & $24-48$ \\
\hline Levofloxacin $5 \mu \mathrm{g}$ & $2(4.8)$ & $40(96.2)$ & $0-28$ \\
\hline Metronidazole $5 \mu \mathrm{g}$ & $42(100)$ & $0(0.00)$ & $32-56$ \\
\hline 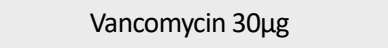 & $42(100)$ & $0(0.00)$ & $20-32$ \\
\hline 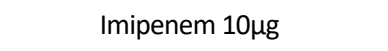 & $8(19)$ & $34(81)$ & $0-32$ \\
\hline Rifampicin $5 \mu \mathrm{g}$ & $42(100)$ & $0(0.00)$ & $16-48$ \\
\hline Azithromycin $15 \mu \mathrm{g}$ & $16(39)$ & $25(61)$ & $0-32$ \\
\hline Erythromycin $15 \mu \mathrm{g}$ & $19(45.2)$ & $23(54.8)$ & $0-40$ \\
\hline Linezolid $30 \mu \mathrm{g}$ & $42(100)$ & $0(0.00)$ & $24-48$ \\
\hline Amoxicillin/clavulanic acid $30 \mu \mathrm{g}$ & $35(83.3)$ & $7(17.7)$ & $20-36$ \\
\hline Chloramphenicol 30 & $32(76)$ & $10(24)$ & $0-34$ \\
\hline
\end{tabular}


Table 2. Prevalence of antibiotic susceptibility by agar dilution method and E-test in C. diff isolates

\begin{tabular}{cccc}
\hline Antibiotic & MIC Range $(\mu \mathrm{g} / \mathrm{ml})$ & Resistant, N0. $(\%)$ & Break Points $(\mu \mathrm{g} / \mathrm{ml})$ \\
\hline Metronidazole & $0.016-0.94$ & $0(0.00)$ & $\geq 32$ \\
Vancomycin & $0.125-0.5$ & $0(0.00)$ & $\geq 2$ \\
Erythromycin & $0.5-16$ & $17(40.5)$ & $\geq 8$ \\
\hline
\end{tabular}

\section{Results}

C. difficile isolates were $100 \%$ resistant to clindamycin; $96.2 \%$ to levofloxacin; $54.8 \%$ to erythromycin; $81 \%$ to imipenem, and $61 \%$ to azithromycin by disk diffusion method. The antibiotic susceptibility of C. diff by disk diffusion method was $95.2 \%$ to teicoplanin; $76 \%$ to chloramphenicol, and $83.3 \%$ to amoxicillin-clavulanic acid. $100 \%$ of the isolates were sensitive to metronidazole, vancomycin, linezolid, tigecycline, and rifampin while $45.2 \%$ were sensitive to erythromycin (Table 1). All C. diff isolates were sensitive to metronidazole using E-test. By agar dilution method, all C. diff isolates were sensitive to vancomycin, while $59.5 \%$ were sensitive to erythromycin (Table 2).

\section{Conclusion}

The present study was performed to determine the susceptibility of $\mathrm{C}$. diff to common antibiotics such as vancomycin, metronidazole and erythromycin in residents of nursing homes in Tehran, Iran. Inappropriate use of antibiotics is one of the effective factors in causing diarrhea by CDI; hence, it is necessary to stop the use of antibiotics for treatment [23]. Antibiotics are one of the most widely used drugs in the world and are extremely valuable in terms of general health. However, the effect of antibiotics in the community and the risk of resistance may also be affected by how the patient uses antibiotics [11]. Antibiotic resistance plays an important role in the emergence of new strains, and transmission of genetic elements can occur between C. diff strains or between C. diff and other bacteria, where increase resistance $[23,38]$.

The results were the same for metronidazole and vancomycin antibiotics by using disk diffusion method, agar dilution method and E-test. C. diff strains were sensitive to metronidazole, vancomycin, linezolid, tigecycline, teicoplanin, rifampin and amoxicillin-clavulanic acid. Therefore, the disk diffusion method can be a suitable method for testing the antibiotic susceptibility of C. diff. Study limitations included financial constraints and difficulty sampling the elderly. Due to our financial constraints, only three metro- nidazole, vancomycin, and erythromycin antibiotics were selected for agar dilution test and E-test.

Clostridium difficile isolates were sensitive to vancomycin and metronidazole antibiotics using disk diffusion and agar dilution methods. These antibiotics can be used in cases of C. diff infection in the elderly. However, the use of vancomycin by injection is not recommended for outpatients. On the other hand, the disk diffusion method, as a simple and inexpensive screening method can be used to determine the antibiotic resistance of $\mathrm{C}$. diff, and resistant isolates be confirmed by Minimum Inhibitory Concentration parameter.

\section{Ethical Considerations}

\section{Compliance with ethical guidelines}

This study was approved by Ethical Committee of Tarbiat Modares University, Tehran.

Funding

This study was supported by Tarbiat Modares University, Tehran and the Research Center for Emerging and Reappearing Infectious Diseases, Pasteur Institute of Iran and also in collaboration with Kahrizak Nursing Home in Tehran.

\section{Authors' contributions}

All authors equally contributed in preparing this article.

\section{Conflicts of interest}

The authors declared no conflict of interest.

\section{Acknowledgements}

The authors would like to thank Dr. Farshad Sharif, Elderly Health Research Center, Tehran University of Medical Sciences. 


\title{
بررسى حساسيت أنتىبيوتيك مؤثر بر كلستريديوم ديفيسيلهاى ايزولهشده از سالمندان مقيم خانه سالمندان در تهران
}

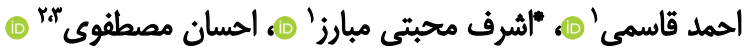 \\ 1. كروه باكترى شناسى، دانشكده هز شكى، دانشعاه تربيت مدرس، تهران، ايران.

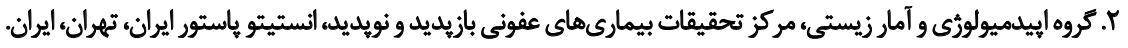

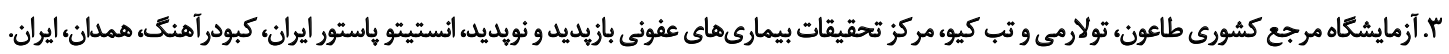

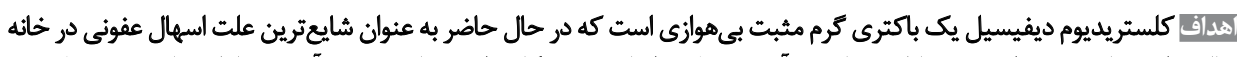

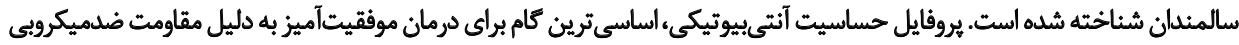

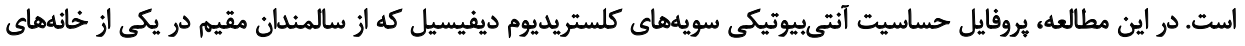
سالمثدان در تهران جدا شده بوده، ارزيابى شدند.

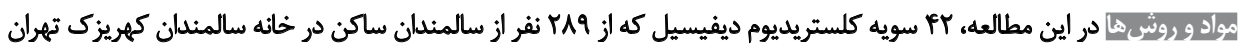

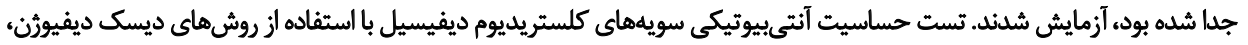

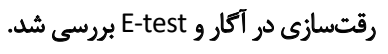

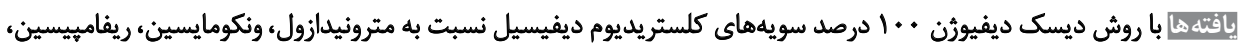

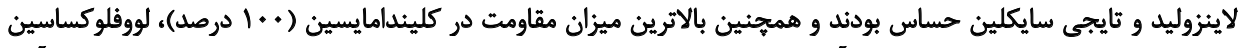

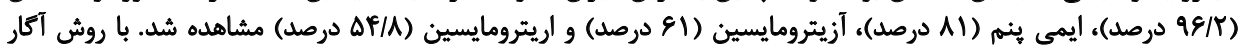

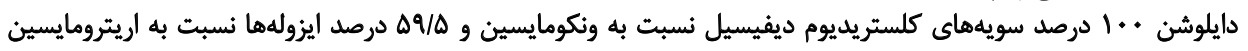

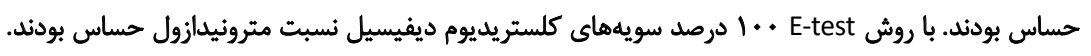

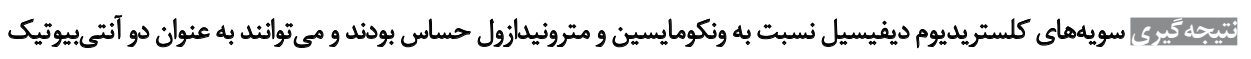

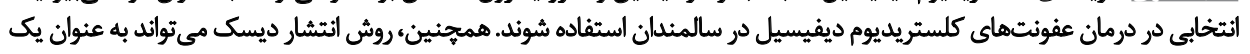
تست غربالكرى براي تعيين مقاومت آنتى بيوتيكى استفاده شون دودي

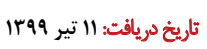

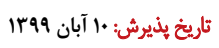

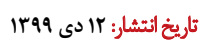

كليدوازوهها: كلستريديوم ديفيسيل، سالمندان، حساسيت آنتى بيوتيكى سالمنياسن

مي كذارد. اين تغييرات اغلب فلور روده را مختل، درنتيجه موجب كسترش كلستريديوم ديفيسيل در روده مي شئود [ب].

dateo

آمارها نشان ميدهد كه اين عارضه بسته به جمعيت و نوع

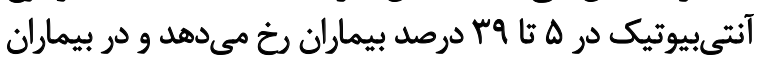

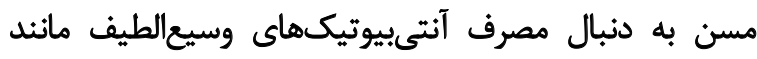

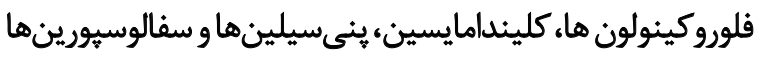
شايعتر است.

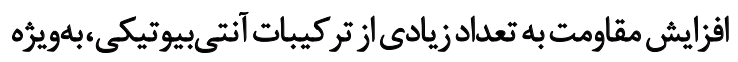

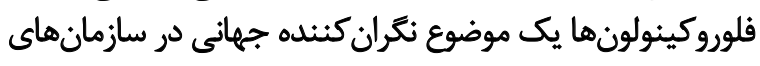

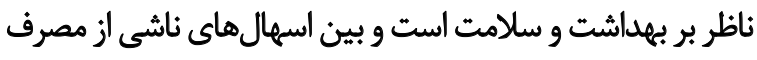

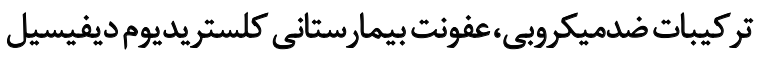

باكترى كلستريديوم ديفيسيل' باسيل كرم مثبت، بىهوازى

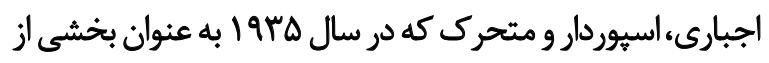

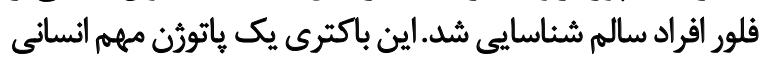

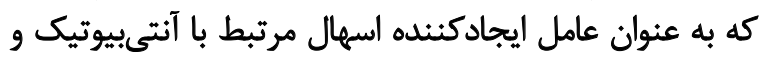

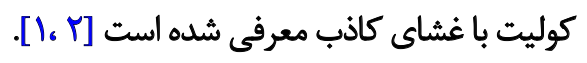

اسهال ناشى از مصرف آنتىبيوتيك، يكى از شايعترين مشكلات

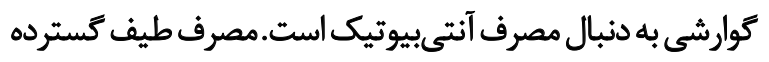
ضدميكروبى كه اثرات مخرب شديدي مئى بر فلور ميكروبى روده

1. Clostridium difficile 
ساكنان خانه سالمندان ايران وجود ندارد. در اين مطالعه، ناقلين

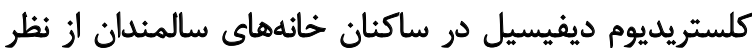
مقاومت آنتىبيوتيكى ارزيابى شدندي درئد dollth نمونهها:در اين مطالعه بافرض • إدرصد آلودتى به كلستريديم

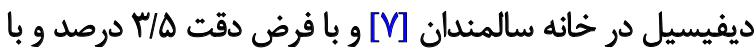

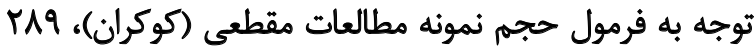

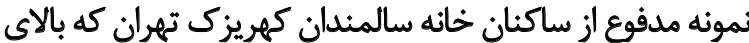

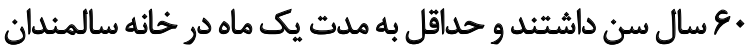
بسترى بودند، از نظر حساسيت آنتىبيوتيكى بررسى شداند.

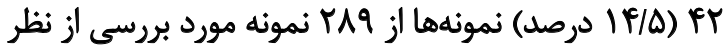

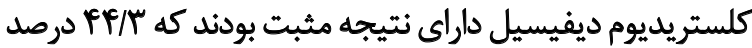

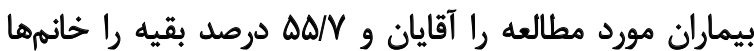

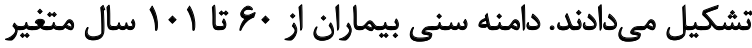

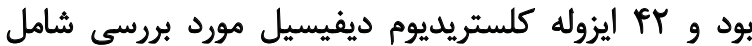

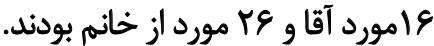

تست حساسيث آنثي بيوتيكي با روش انتشار ديسك ديسك آنتىبيوتيكهاى مورد بررسى مترونيدازول،

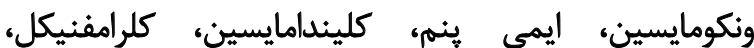

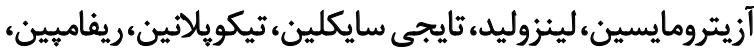

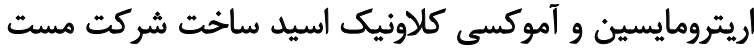

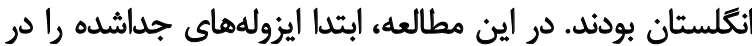

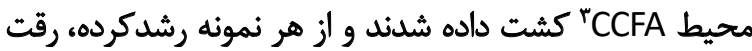
يكا مك فارلند در سرم فيزيولوثى تهيه شد. سيس توسط سواب روى محيط بروسلا آتار (ساخت شركت

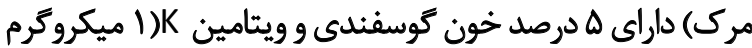

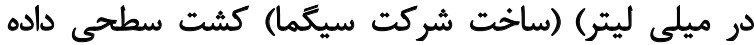

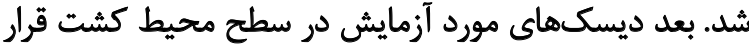

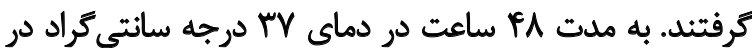

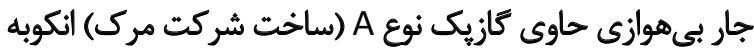

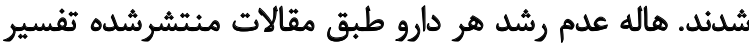

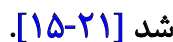

\section{PE-test تست حساسيت أتثى بيوتيكي با ووش آتاردايلوشن}

تست حساسيت آنتىبيوتيكى با روش آكار دايلوشن براى آنتىبيوتيكهاي ونكومايسين و اريترومايسين ساخت ريت شركت

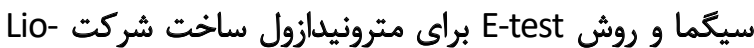
f ايتاليا، طبق بروتكل Elilchem

3. Cycloserine-Cefoxitin Fructose Agar

4. Epsilometer test

5. Clinical and Laboratory Standards Institute 2018

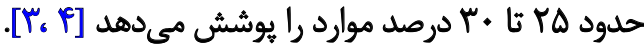
كلستريديوم ديفيسيل به عنوان شايعترين عامل اسهال عفونى

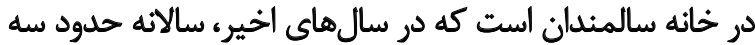

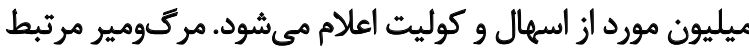

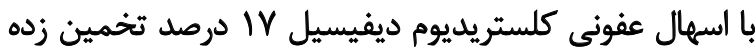

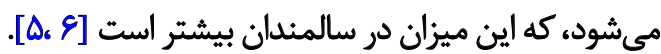
اسهال مكرر مرتبط با كلستريديوم ديفيسيل بيشتر در ساكنان

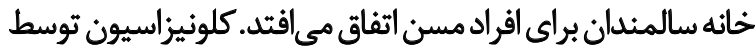

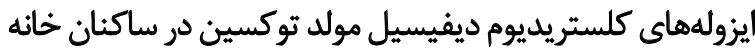

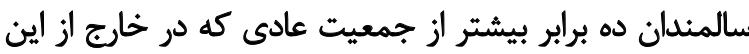

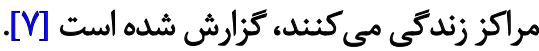
مطالعات نشان ميدهد كه وضعيت جسمى ساكنان خانه

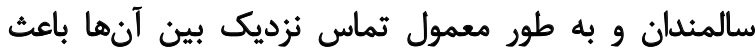

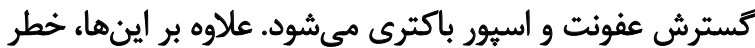

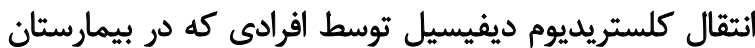

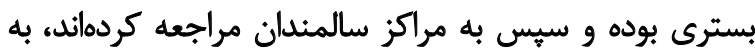
عنوان يك منبع و ناقل بالقوه آلودئى است.

كزارش شده كه حدود QV د درصد از ساكنان خانه سالمندان

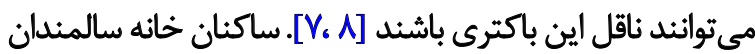

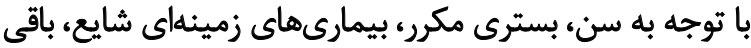

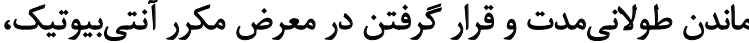

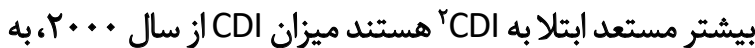

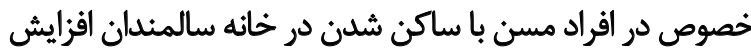
يافته است [• [-1-1].

درمان آنتىبيوتيكى نقش اصلى راد دي بيشرفت CDI دارد. اكر

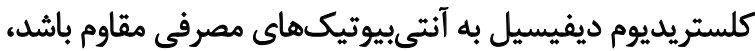

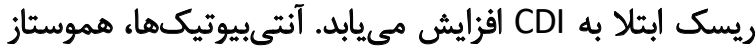

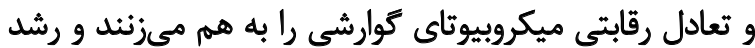

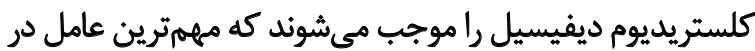

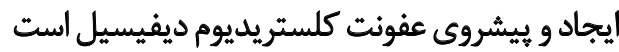
مصرف همزمان جندين آنتىبيوتيك وافزايش طول مدت درمان

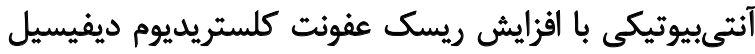

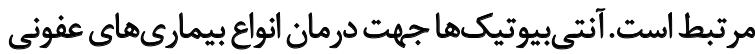

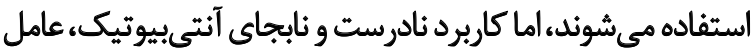

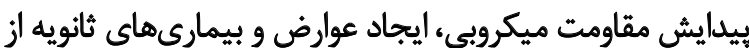

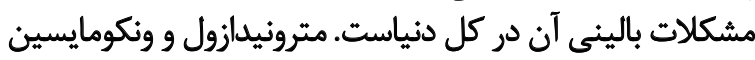

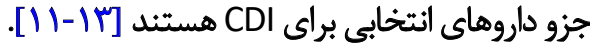

در كشور ما نيز معضل مصرف غيرمنطقى و بيش از حدا حد

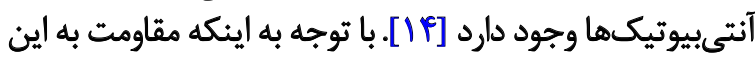
داروها در دنيا رو به افزايش است و هيج كزارشى در اين مورد در

2. Clostridium Difficile Infection 
يافتهها

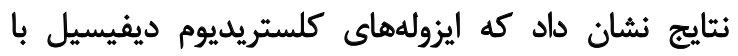

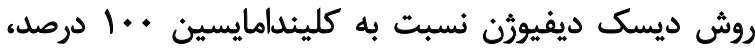

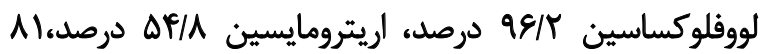

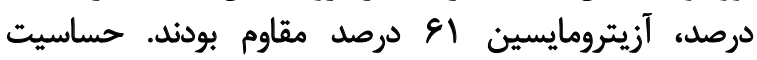

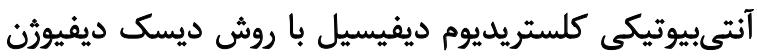

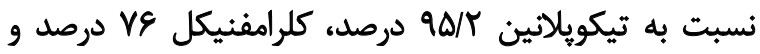

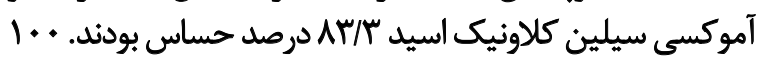

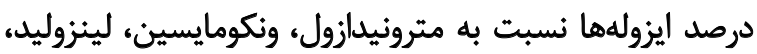

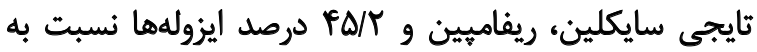
اريترومايسين حساس بودند (جدول شماره (1).

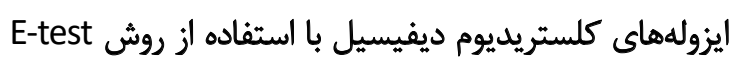

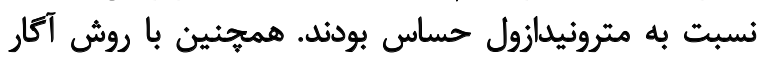

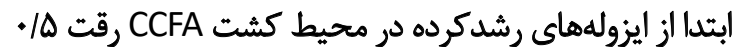

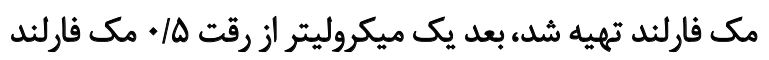

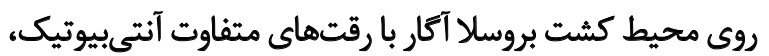

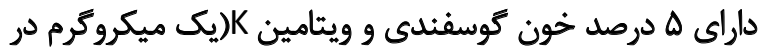

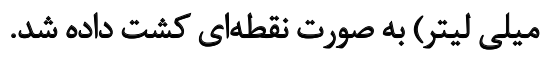

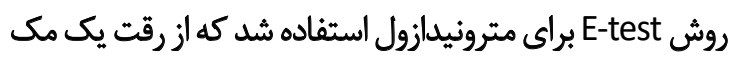

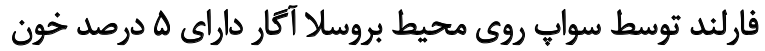

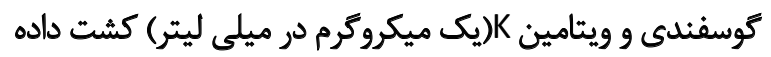

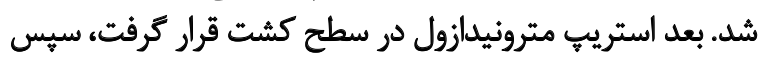

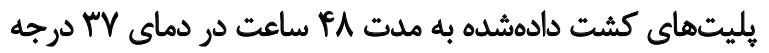

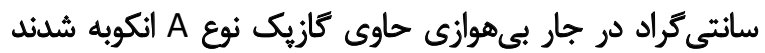

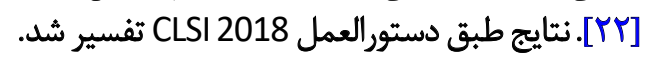

جدول ا. فراوانى حساسيت آنتىبيوتيكى با روش ديسك ديفيورن در ايزولههاى كلستريديوم ديفيسيل جداشده از سالمندان

\begin{tabular}{|c|c|c|c|}
\hline \multirow{2}{*}{ زون كسترشي } & \multicolumn{2}{|c|}{ No. (\%) } & \multirow{2}{*}{ أنتىبيوتيك (ميلى كُرم) } \\
\hline & مقاومت & حساسيت & \\
\hline$\cdot$ & or $(1.0)$ & $\cdot(\cdot / *)$ & كليندامايسين Y \\
\hline$M r-19$ & $r(r / \lambda)$ & $P \cdot(q \Delta / r)$ & تيكويلانين • r \\
\hline PA-TF & $\cdot(\cdot 1 \cdot \cdot)$ & er $(1.0)$ & تايجي سايكلين 10 \\
\hline TA-. & $f \cdot(98 / \pi)$ & $r(f / A)$ & لووفلوكساسين ه \\
\hline$\Delta s-m$ & $\cdot(\cdot 1 \cdot \cdot)$ & $P r(1+\cdots)$ & هترونيدازول ه ه \\
\hline Mr-r. & $\cdot(\cdot 1 \cdot \cdot)$ & Pr $(1 \cdots)$ & 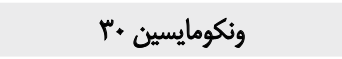 \\
\hline$M-\cdot$ & $m f(\lambda))$ & $\wedge(19)$ & ايمى ينه ·1 \\
\hline$P A-1 E$ & $+(+1++)$ & $\mu r(1+*)$ & ريفاميين ه \\
\hline$M-$. & $r \Delta(8)$ & $18(19)$ & آزيترومايسين 10 \\
\hline r.-. & $M(\Delta F / A)$ & $19(F \Delta / Y)$ & اريترومايسين 10 \\
\hline PA-MP & $\cdot(\cdot 1 \cdot \cdot)$ & er $(1.0)$ & لينزوليد +ب \\
\hline$r q-Y$. & $V(I V / V)$ & $\Delta(A T / M)$ & آموكسى سيلين/كلاونيك اسيد •ب \\
\hline$m f-$. & $1 \cdot(\mathrm{MY})$ & $\Pi(V E)$ & كلرامفنيكل +r r \\
\hline
\end{tabular}

L

جدول r. فراوانى حساسيت آنتىبيوتيكى با روش آكار دايلوشن و E-test در كلستريديوم ديفيسيل

\begin{tabular}{|c|c|c|c|}
\hline فواصل مصرف دالرو (Mg/ml) & حسايت تعداد (درصد) & ( دامنه & أثتى ييوتيك \\
\hline$\geq m$ & $\cdot(\cdot 1 \cdot)$. & $.1 .18-. / 97$ & مثرونيلازول \\
\hline$\geq Y$ & $\cdot(\cdot 1 \cdot \cdot)$ & $. / 1150-10$ & ونكومايسين \\
\hline$\geq \wedge$ & $\operatorname{IV}(f+/ \Delta)$ & $+10-+118$ & الريترومايسين \\
\hline
\end{tabular}

L 


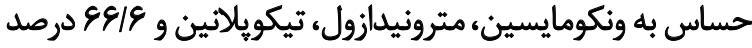

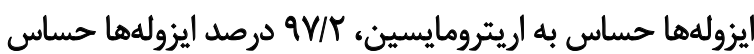

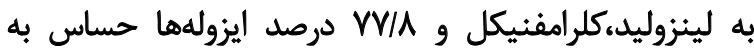

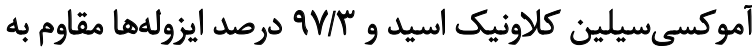

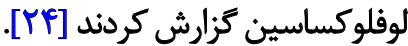

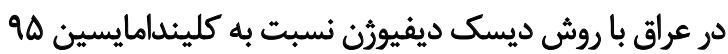

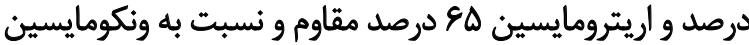

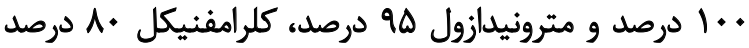

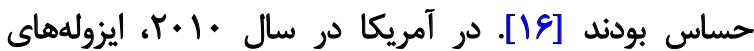

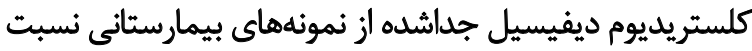

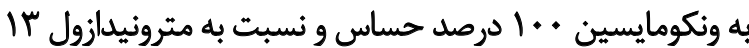
درصد مقاوم بودند [• [ب].

الكوى حساسيت آنتىبيوتيكى ايزولههاى كلستريديوم

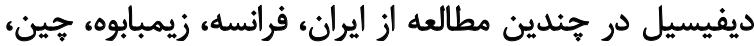

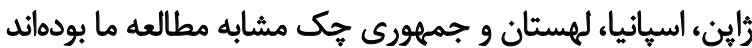

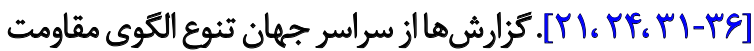
رابراى ايزولههاى كلستريديوم ديفيسيل بيشنهادئنمى كنيند. در مطالعه حاضر، همه سويهها حساس به ونكومايسين

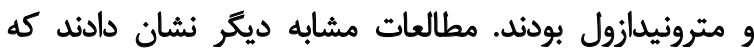

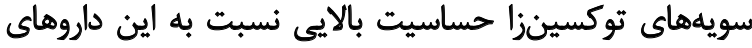

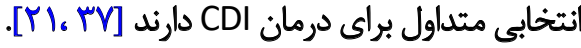

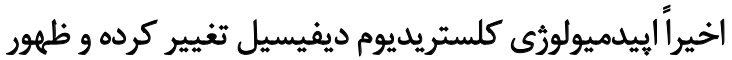

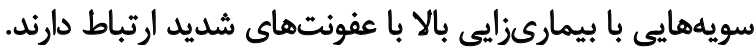

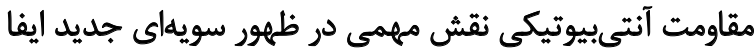

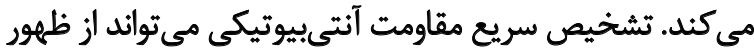
فنوتييى جديد جلوكيرى كرده و به شناسايى سويههاى جديد انديد

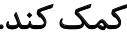

رثنوم كلستريديوم ديفيسيل حاوى عناصر رنتيكيى است كه

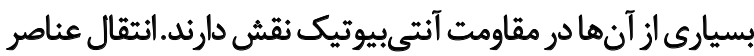

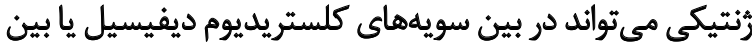

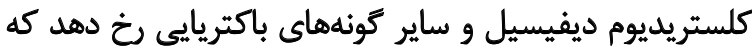

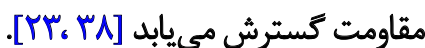
نتايج با روش ديسك ديفيوزن، آكار دايلوشن و E-test براى

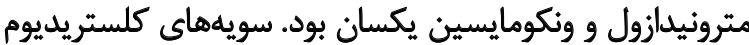

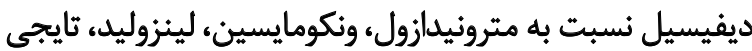

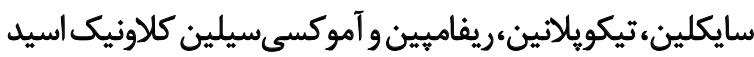

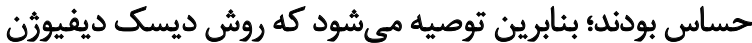

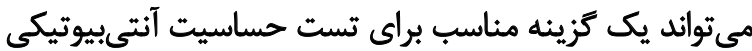

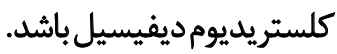

محدوديتهاى مطالعه شامل كمبود منبع مالى و مشكل بـ مالي نمونهكيرى از سالمندان بود. با توجه به محدوديت منديت مالى مالى كه مثلى
دايلوشن، همه ايزولههاى كلستريديوم ديفيسيل نسبت به به ونكومايسين حساس بودند، ولى هو/ه درصد به به اريترومايسين حساس بودند (جدول شماره ج)

4

مطالعه حاضر براى تعيين حساسيت كلستريديوم ديفيسيل

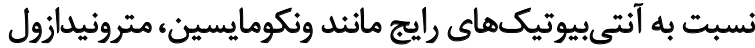
و اريترومايسين در ساكنان خانه سالمندان تهران انجام كرفت مصرف بكرويه آنتىبيوتيكها يكى از عوامل مؤثر در ايجاد اسهال توسط CDI است كه قطع كردن مصرف آنتىبيونيك آنتيك

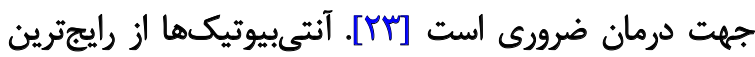
داروهاى مصرفى در سراسر جهان و از نظر سلامت إنى عمومى داراي

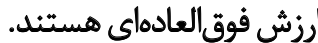

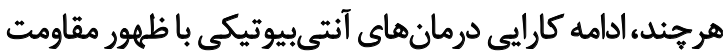

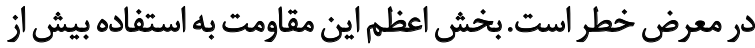

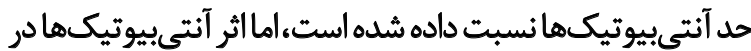

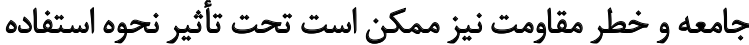

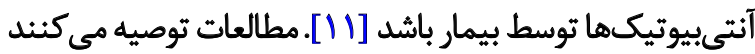

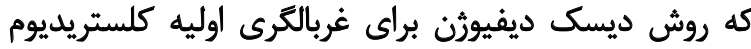

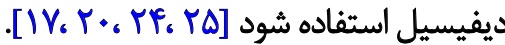

در اين مطالعه نتايج نشان داد كه ايزولههاي كلستريديوم

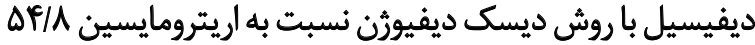

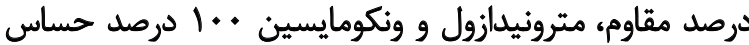

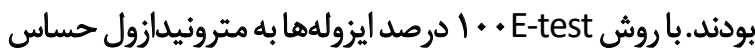

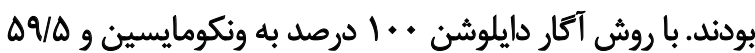

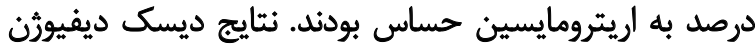
مطابق با نتايج آكار دايلوشن و E-test بودين مطالعايى در تبريز با روش ديسك ديفيوثن • .1 درصد

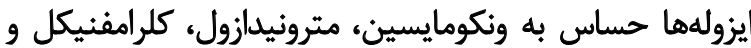

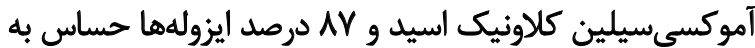

اريترومايسين بودند [1هائي كلاونيكي

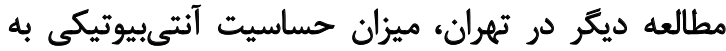

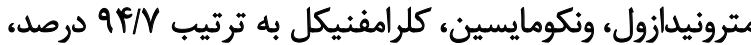

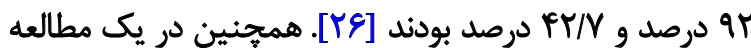

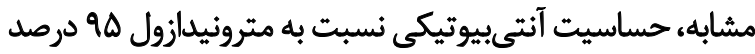

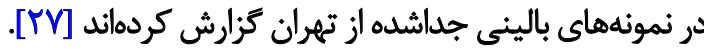

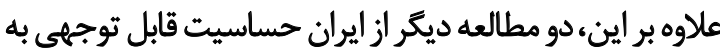

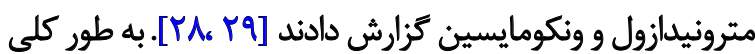

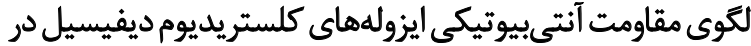
كزارشهاي فوق از ايران مشابه بودهاند. در فرانسه با روش ديسك ديفيوزن ..1 درصد ايزولهها 
داشتيم براى انجام تست آكار دايلوشن و E-test سه آنتىبيوتيك

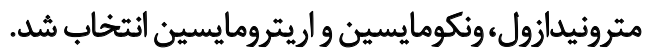

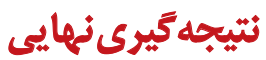

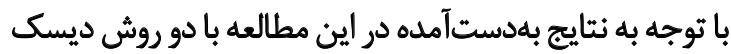

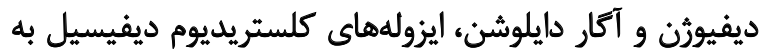

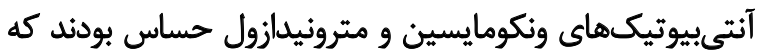

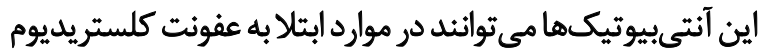
ديفيسيل در سالمندان استفاده شوند.

با وجود اين استفاده از ونكومايسين تزريقى (به علت تزريقى

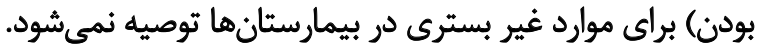

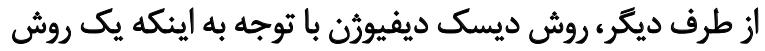

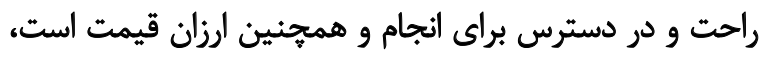

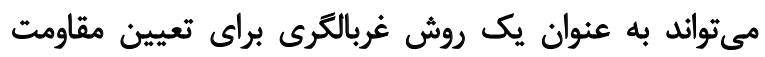

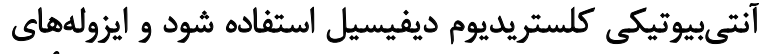
مقاوم با روش

شود.

مالاجظات اخلاقي

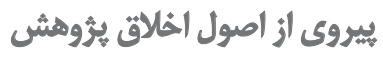

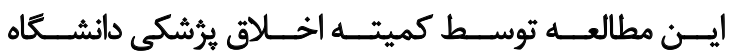

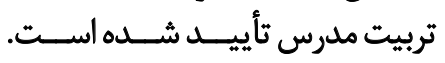

مامي مالب

اين برثوهش با حمايت مالى دانشغاه تربيت مدرس، تمبران و و

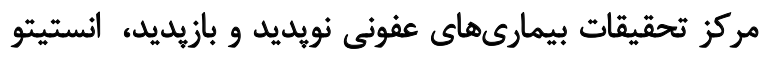

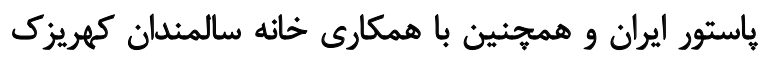

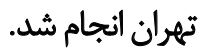

$$
\text { مشاركت نويسيندان }
$$

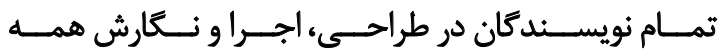

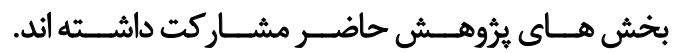

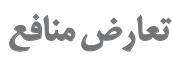

نويسندكان العلام مي كنئد كه هيج كونه تعارض منافع در اين مطالعهidi

$$
\text { تشكير وقدر مانى }
$$

بدين وسيله نويسندكان از دكتر فرشاد شريف، مركز تحقيقات

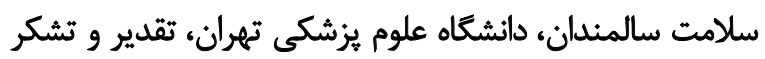
مي كنيند. 


\section{References}

[1] Smits WK, Lyras D, Lacy DB, Wilcox MH, Kuijper EJ. Clostridium difficile infection. Nature Reviews Disease Primers. 2016; 2:16020. [DOI:10.1038/nrdp.2016.20] [PMID] [PMCID]

[2] Rodriguez C, Taminiau B, Korsak N, Avesani V, Van Broeck J, Brach P, et al. Longitudinal survey of Clostridium difficile presence and gut microbiota composition in a Belgian nursing home. BMC Microbiology. 2016; 16:229. [DOI:10.1186/s12866016-0848-7] [PMID] [PMCID]

[3] Vincent C, Manges AR. Antimicrobial use, human gut microbiota and Clostridium difficile colonization and infection. Antibiotics. 2015; 4(3):230-53. [DOI:10.3390/antibiotics4030230] [PMID] [PMCID]

[4] Esfandiari Z, Jalali M, Safaeian L, Weese JS. [A review on epidemiology of Clostridium difficile infection (Persian)]. Tehran University Medical Journal TUMS Publications. 2016; 74(5):305-13. http:/ / tumj.tums.ac.ir/article-1-7573-en.html

[5] Hunter JC, Mu Y, Dumyati GK, Farley MM, Winston LG, Johnston HL, et al. Burden of nursing home-onset Clostridium difficile infection in the United States: Estimates of incidence and patient outcomes. Open Forum Infectious Diseases. 2016; 3(1). [DOI:10.1093/ofid/ofv196] [PMID] [PMCID]

[6] Crogan NL, Evans BC. Clostridium difficile: An emerging epidemic in nursing homes. Geriatric Nursing. 2007; 28(3):1614. [DOI:10.1016/j.gerinurse.2007.04.005] [PMID]

[7] Rodriguez C, Korsak N, Taminiau B, Avesani V, Van Broeck J, Delmée $\mathrm{M}$, et al. Clostridium difficile infection in elderly nursing home residents. Anaerobe. 2014; 30:184-7. [DOI:10.1016/j. anaerobe.2014.08.007] [PMID]

[8] Surawicz CM, Brandt LJ, Binion DG, Ananthakrishnan AN, Curry SR, Gilligan PH, et al. Guidelines for diagnosis, treatment, and prevention ofclostridium difficileInfections. American Journal of Gastroenterology. 2013; 108(4):478-98. [DOI:10.1038/ajg.2013.4] [PMID]

[9] Fuchs BB, Tharmalingam N, Mylonakis E. Vulnerability of long-term care facility residents to Clostridium difficile infection due to microbiome disruptions. Future Microbiology. 2018; 13(13):1537-47. [DOI:10.2217/fmb-2018-0157] [PMID]

[10] Yu H, Baser O, Wang L. Burden of Clostridium difficileassociated disease among patients residing in nursing homes: A population-based cohort study. BMC Geriatrics. 2016; 16(1):193. [DOI:10.1186/s12877-016-0367-2] [PMID] [PMCID]

[11] Kardas P, Devine S, Golembesky A, Roberts C. A systematic review and meta-analysis of misuse of antibiotic therapies in the community. International Journal of Antimicrobial Agents. 2005; 26(2):106-13. [DOI:10.1016/j.ijantimicag.2005.04.017] [PMID]

[12] Peng Z, Jin D, Kim HB, Stratton CW, Wu B, Tang YW, et al. Update on antimicrobial resistance in Clostridium difficile: resistance mechanisms and antimicrobial susceptibility testing. Journal of Clinical Microbiology. 2017; 55(7):1998-2008. [DOI:10.1128/JCM.02250-16] [PMID] [PMCID]

[13] Monaghan T, Boswell T, Mahida YR. Recent advances in Clostridium difficile-associated disease. Postgraduate Medical Journal. 2009; 85(1001):152-62. [DOI:10.1136/gut.2007.128157]
[14] Hadi A, Mohammad H, Alireza Y, Saman Z. Antibiotic utilization in Iran 2000-2016: Pattern analysis and benchmarking with organization for economic co-operation and development countries. Journal of Research in Pharmacy Practice. 2019; 8(3):162-7. [DOI:10.4103/jrpp.JRPP_19_42] [PMID] [PMCID]

[15] Akhi MT, Pirzade T, Naghili B, Gojazade M. Antimicrobial susceptibility of Clostridium difficile isolated from different sources of Imam Reza Hospital, Tabriz. African Journal of Microbiology Research. 2011; 5(19):2946-9. [DOI:10.5897/AJMR11.423]

[16] Mehdi LY, AL-Mossawei MT. Antibiotic susceptibility testing for Clostridium difficile Iraqi isolation by using disk diffusion method. Journal of Biology, Agriculture and Healthcare. 2015; 5(13):156-62. https://www.iiste.org/Journals/index.php/ $\mathrm{JBAH} /$ article/view/23964

[17] Fraga EG, Nicodemo AC, Sampaio JLM. Antimicrobial susceptibility of Brazilian Clostridium difficile strains determined by agar dilution and disk diffusion. The Brazilian Journal of Infectious Diseases. 2016; 20(5):476-81. [DOI:10.1016/j.bjid.2016.07.004] [PMID]

[18] Levett P. Antimicrobial susceptibility of Clostridium difficile determined by disc diffusion and breakpoint methods. Journal of Antimicrobial Chemotherapy. 1988; 22(2):167-73. [DOI:10.1093/ jac/22.2.167] [PMID]

[19] Nagy E, Justesen US, Eitel Z, Urbán E. Development of EUCAST disk diffusion method for susceptibility testing of the Bacteroides fragilis group isolates. Anaerobe. 2015; 31:65-71 [DOI:10.1016/j.anaerobe.2014.10.008] [PMID]

[20] Erikstrup LT, Danielsen T, Hall V, Olsen K, Kristensen B, Kahlmeter G, et al. Antimicrobial susceptibility testing of Clostridium difficile using EUCAST epidemiological cut-off values and disk diffusion correlates. Clinical Microbiology and Infection. 2012 18(8):E266-72. [DOI:10.1111/j.1469-0691.2012.03907.x] [PMID]

[21] Shoaei P, Shojaei H, Jalali M, Khorvash F, Hosseini SM, Ataei B, et al. Clostridium difficile isolated from faecal samples in patients with ulcerative colitis. BMC Infectious Diseases. 2019; 19:361. [DOI:10.1186/s12879-019-3965-8] [PMID] [PMCID]

[22] Weinstein MP, Patel JB, Campeau S, Eliopoulos GM, Galas MF, Humphries RM, et al. Performance standards for antimicrobial susceptibility testing. Clinical and Laboratory Standards Institute. 2018; 38.

[23] Spigaglia P. Recent advances in the understanding of antibiotic resistance in Clostridium difficile infection. Therapeutic Advances in Infectious Disease. 2016; 3(1):23-42. [DOI:10.1177/2049936115622891] [PMID] [PMCID]

[24] Poilane I, Bert F, Cruaud P, Nicolas-Chanoine M, Collignon A. Interest of the disk diffusion method for screening Clostridium difficile isolates with decreased susceptibility to antibiotics. Pathologie-Biologie. 2007; 55(8-9):429-33. [DOI:10.1016/j.patbio.2007.07.010] [PMID]

[25] Huang H, Weintraub A, Fang H, Nord CE. Antimicrobia resistance in Clostridium difficile. International Journal of Antimicrobial Agents. 2009; 34(6):516-22. [DOI:10.1016/j.ijantimicag.2009.09.012] [PMID]

[26] Goudarzi M, Goudarzi H, Alebouyeh M, Rad MA, Mehr FSS, Zali MR, et al. Antimicrobial susceptibility of Clostridium difficile clinical isolates in Iran. Iranian Red Crescent Medical Journal. 2013; 15(8):704-11. [DOI:10.5812/ircmj.5189] [PMID] [PMCID] 
[27] Shayganmehr FS, Alebouyeh M, Azimirad M, Aslani MM, Zali MR. Association of tcdA+/tcdB+Clostridium difficile genotype with emergence of multidrug-resistant strains conferring metronidazole resistant phenotype. Iranian Biomedical Journal. 2015; 19(3):143-8. [DOI: 10.7508/ibj.2015.03.003]

[28] Sadeghifard N, Salari MH, Ghassemi MR, Eshraghi S, Harati FA. The incidence of nosocomial toxigenic Clostridium difficile associated diarrhea in Tehran tertiary medical centers. Acta Medica Iranica. 2010; 48(5):320-5. https://acta.tums.ac.ir/index.php/ acta/article/view/4057

[29] Shoaei P, Shojaei H, Khorvash F, Hosseini SM, Ataei B, Tavakoli $\mathrm{H}$, et al. Molecular epidemiology of Clostridium difficile infection in Iranian hospitals. Antimicrobial Resistance \& Infection Control. 2019; 8:12. [DOI:10.1186/s13756-018-0454-6] [PMID] [PMCID]

[30] Norman KN, Scott HM, Harvey RB, Norby B, Hume ME. Comparison of antimicrobial susceptibility among Clostridium difficile isolated from an integrated human and swine population in Texas. Foodborne Pathogens and Disease. 2014; 11(4):257-64. [DOI:10.1089/fpd.2013.1648] [PMID]

[31] Simango C, Uladi S. Detection of clostridium difficile diarrhoea in Harare, Zimbabwe. Transactions of The Royal Society of Tropical Medicine and Hygiene. 2014; 108(6):354-7. [DOI:10.1093/ trstmh/tru042] [PMID]

[32] Oka K, Osaki T, Hanawa T, Kurata S, Okazaki M, Manzoku T, et al. Molecular and microbiological characterization of Clostridium difficile isolates from single, relapse, and reinfection cases. Journal of Clinical Microbiology. 2012; 50(3):915-21. [DOI:10.1128/ JCM.05588-11] [PMID] [PMCID]

[33] Rodríguez-Pardo D, Almirante B, Bartolomé RM, Pomar V, Mirelis B, Navarro F, et al. Epidemiology of Clostridium difficile infection and risk factors for unfavorable clinical outcomes: Results of a hospital-based study in Barcelona, Spain. Journal of Clinical Microbiology. 2013; 51(5):1465-73. [DOI:10.1128/ JCM.03352-12] [PMID] [PMCID]

[34] Obuch-Woszczatyński P, Lachowicz D, Schneider A, Mól A, Pawłowska J, Ożdżeńska-Milke E, et al. Occurrence of Clostridium difficile PCR-ribotype 027 and it's closely related PCR-ribotype 176 in hospitals in Poland in 2008-2010. Anaerobe. 2014; 28:13-7. [DOI:10.1016/j.anaerobe.2014.04.007] [PMID]

[35] Krutova M, Matejkova J, Tkadlec J, Nyc O. Antibiotic profiling of Clostridium difficile ribotype 176-a multidrug resistant relative to C. difficile ribotype 027. Anaerobe. 2015; 36:88-90. [DOI:10.1016/j.anaerobe.2015.07.009] [PMID]

[36] Tian T-t, Zhao J-h, Yang J, Qiang C-x, Li Z-r, Chen J, et al. Molecular characterization of Clostridium difficile isolates from human subjects and the environment. PloS One. 2016; 11(3):e0151964. [DOI: 10.1371/journal.pone.0151964]

[37] Ngamskulrungroj P, Sanmee S, Pusathit P, Piewngam P, Elliott $\mathrm{B}$, Riley TV, et al. Molecular epidemiology of Clostridium difficile infection in a large teaching hospital in Thailand. PloS One. 2015; 10(5):e0127026. [DOI:10.1371/journal.pone.0127026] [PMID] [PMCID]

[38] Johanesen PA, Mackin KE, Hutton ML, Awad MM, Larcombe $\mathrm{S}$, Amy JM, et al. Disruption of the gut microbiome: Clostridium difficile infection and the threat of antibiotic resistance. Genes. 2015; 6(4):1347-60. [DOI:10.3390/genes6041347] [PMID] [PMCID] 